ECMO, both of whom survived to discharge as did all babies who underwent surgical repair of the $\mathrm{CDH}$.

Conclusion There was an $11.1 \%$ mortality rate increase amongst the cohort studied when compared with the preceding 5 year block. A notably lower termination rate (28\% vs. 50\%) could possibly account for this, in addition to associated anomalies as above. Variations in management approach within the team was observed leading to the subsequent formulation of an evidence based protocol to improve care quality and future outcomes as current evidence suggests.

\section{PS-297a CATCH-UP-GROWTH IN TERM AND PRETERM INFANTS AFTER SURGICAL CLOSURE OF VENTRICULAR SEPTAL DEFECT IN THE FIRST YEAR OF LIFE: ONLY GOOD NEWS?}

${ }^{1} \mathrm{~L}$ Martins, ${ }^{1} \mathrm{R}$ Lourenço, ${ }^{2} \mathrm{~S}$ Cordeiro, ${ }^{2} \mathrm{~N}$ Carvalho, ${ }^{3} \mathrm{M}$ Loureiro, ${ }^{3} \mathrm{M}$ Patrício, ${ }^{2} \mathrm{R}$ Anjos. ${ }^{1}$ Department of Pediatrics, Hospital Do Divino Espirito Santo de Ponta Delgada EPE, Ponta Delgada, Portugal; ' 2 Department of Pediatric Cardiology, Hospital de Santa Cruz Centro Hospitalar de Lisboa Ocidental EPE, Lisbon, Portugal; ${ }^{3}$ Laboratory of Biostatistics and Medical Informatics IBILI, Faculty of Medicine of University of Coimbra, Coimbra, Portugal

\subsection{6/archdischild-2014-307384.596}

Failure to thrive is common in children with non-restrictive ventricular septal defect (VSD). Normalisation of growth has been reported after early surgical correction. However the literature is inconsistent about growth velocity after surgery in term and preterm infants.

Objective Establishing the pattern of catch-up growth in infants submitted to VSD surgical repair before 1 year of age, for term and preterm infants.

Methods 52 infants (41 term, 11 preterm) were studied. Anthropometric data at birth, at time of surgery and 3, 6, 12 and 24 months after surgery, collected retrospectively, were converted to z-scores. Statistic analyses was performed in SPSS ${ }^{\circledR}$ version 21, $\alpha=0,05$.

Results Mean weight and height $\mathrm{z}$ scores at the time of surgery were significantly lower for term infants $(-2,24$ and $-1,42$, respectively; $\mathrm{p}<0,001)$ and preterm infants $(-3,07$ and $-2,22 ; \mathrm{p}$ $=0,003)$. A higher growth velocity was observed in the first three months after surgery. For term infants, catch-up growth was completed 6 months after surgery (mean weight and height z scores were $-0,39$ and $-0,7$, respectively). Preterm infants completed their catch up growth one year after surgery. There were no statistically significant differences in mean weight and height between term and preterm infants 24 month after surgery.

Conclusions Early surgical repair of VSD leads to a significant acceleration of growth, mainly in the first 3-6 months after surgery. An increased weight gain velocity has been associated with higher cardiovascular risk later in life. Knowledge of this specific
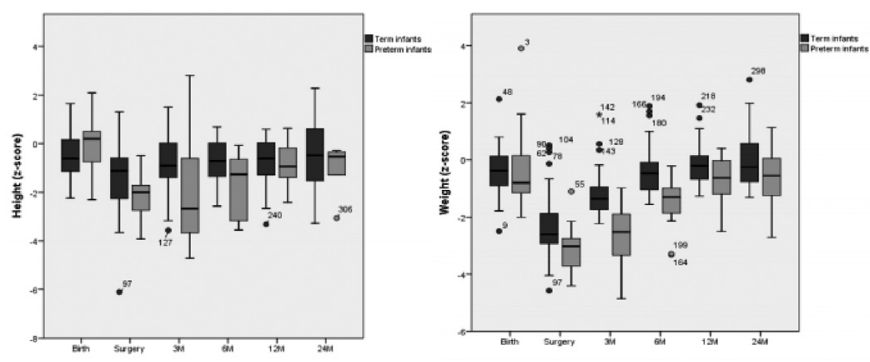

Abstract PS-297a Figure 1 catch-up growth pattern is important and should influence nutritional goals after surgery.

\section{Perinatal Infection}

\section{PS-298 RANDOMISED CONTROLLED TRIAL TO COMPARE EFFICACY OF DIFFERENT TIMING OF ANTIBIOTICS AT CAESAREAN SECTION AND THEIR EFFECT ON MOTHER AND NEWBORN}

${ }^{1}$ KA Kuruvilla, ${ }^{1} \mathrm{CA}$ Jyothirmayi, ${ }^{2} \mathrm{~A}$ Halder, ${ }^{2} \mathrm{R}$ Jose, ${ }^{1} \mathrm{~S}$ Sridhar. ${ }^{1} \mathrm{C}$ hild Health and Neonatology, Christian Medical College, Vellore, India; ${ }^{2}$ Obstetrics and Gynaecology, Christian Medical College, Vellore, India

\subsection{6/archdischild-2014-307384.597}

Background During caesarean section, prophylactic antibiotics are usually given after cord clamping (instead of prior to skin incision) for fear of antibiotics having adverse effects on the newborn and promoting resistant strains.

Objective To compare efficacy of intravenous cefazoline administered during caesarean section either before skin incision or after cord clamping, on mother and newborn.

Setting Tertiary care perinatal centre in south India.

Methods Term gestation mothers posted for caesarean section were randomised to receive two medicinesIV cefazoline/ placeboone prior to skin incision, one after cord clamping. Mothers and babies were monitored for evidence of infection or adverse events during hospital stay. They were reviewed at 45 days to look for complications.

Results 1106 mothers were recruited. At baseline, mothers and babies in both groups were similar. The mean (SD) duration of hospital stay for mothers in both groups was 5.3(1.5) days. Mothers who received antibiotics prior to skin incision had less post operative complications compared to mothers who received antibiotics after cord clamping $(p=0.000)$. Mothers who received antibiotics after cord clamping stayed longer in hospital $(\mathrm{p}=0.008)$. Babies in both groups had similar rates of nursery admissions, sepsis, NEC and hospital readmission following discharge.

Conclusions IV antibiotics can be safely administered to mothers prior to skin incision which decreases postoperative infectious morbidity without adverse effects in babies.

\section{PS-299 HAEMATOLOGICAL MARKERS OF VERTICAL TRANSMISSION OF GENITAL MYCOPLASMAS IN PREMATURE INFANTS}

${ }^{1} \mathrm{~N}$ Hussain, ${ }^{2} \mathrm{~L}$ Rahman. 'Pediatrics, Connecticut Children's Medical Center, Hartford, USA; ${ }^{2}$ Pediatrics, University of Connecticut Health Center, Farmington, USA

\subsection{6/archdischild-2014-307384.598}

Background Genital mycoplasmas (Ureaplasma urealyticum-Uu or Mycoplasma hominis - Mh) are low grade pathogens associated with complications of pregnancy (chorioamnionitis and preterm labour); but their role as neonatal pathogens is controversial.

Aim To identify haematological markers of vertical transmission of genital mycoplasma in premature newborn infants.

Methods A retrospective cohort study done at University of Connecticut Health Centre NICU with admissions from 20032010. Intubated infants in the NICU had tracheal cultures sent 